\section{Capsaicin Content of West Indies Hot Pepper Cultivars Using Colorimetric and Chromatographic Techniques}

\author{
Harold A.A. Gibbs ${ }^{1}$ and Leonard W. O'Garro \\ DepartmentofBiological and Chemical Sciences, Cave HillCampus, University \\ of the West Indies, Barbados
}

\section{Additional index words. Capsicum chinense, pungency, variety, colorimetric, HPLC}

\begin{abstract}
Colorimetric and chromatographic methods were used to assess capsaicinoid levels in a pungent Caribbean-grown pepper collection comprising 28 accessions of Capsicum chinense and one each of $C$. annuum and $C$. frutescens. Two colorimetric methods, one commonly used and attributed to Bajaj (1980) and a modification of the Bajaj method were also compared for congruity and ease of use. Capsaicin content of the cultivars ranged from 37.6 to $497.0 \mathrm{mg} / 100 \mathrm{~g}$ in ripe fruits and 27.8 to $404.5 \mathrm{mg} / 100 \mathrm{~g}$ in green fruit, as determined by Bajaj's method. The corresponding Scoville units of pungency varied from 15,000 to 300,000 for ripe fruit and 7,500 to 270,000 for green fruit. Levels of capsaicin assessed by the modified Bajaj method varied from 15.0 to $402.4 \mathrm{mg} / 100 \mathrm{~g}$ and 13.7 to 356.4 $\mathrm{mg} / 100 \mathrm{~g}$ in ripe and green fruit, respectively. On the basis of capsaicin levels assessed by each colorimetric method, the pepper cultivars were differentiated into seven distinct pungency groups. For each method, similar groupings of cultivars were observed for ripe and green fruit and groups of the same numerical designation were mainly comprised of common assessions. These results indicate that the two colorimetric methods generally agree. In contrast, the modified colorimetric method was more efficient than Bajaj's procedure, which required pretreatment of pepper extracts to remove the extracting solvent by evaporation and interfering chromogenic pigments by column chromatography. Phase separation of capsaicin and interfering pigments in pepper extracts by use of dilute acid was the only pretreatment required in the modified Bajaj method before colorimetry. High performance liquid chromatography performed on fruit extracts of the cultivars revealed the presence of the capsaicinoids capsaicin, homocapsaicin, dihydrocapsaicin, nordihydrocapsaicin, and homodihydrocapsaicin. Capsaicin and homocapsaicin were detected in greater abundance than dihydrocapsaicin and nordihydrocapsaicin in fruit of all cultivars. Homodihydrocapsaicin was the least abundant of the capsaicinoids and was generally absent in ripe fruit.
\end{abstract}

A rich diversity of pungent peppers is grown in the Caribbean (Greenleaf, 1985). These peppers have attracted attention in light of increasing demand for pungent peppers as food condiments in North America and Europe. However, with the exception of the Capsicum chinense cultivars 'Scotch Bonnet' and 'West Indies Red', pungent peppers of the Caribbean are marginal or under-exploited crops. As a consequence, there is little or no published information on pungency of $C$. chinense grown in the Caribbean.

Pungency in peppers is due to the amount of capsaicinoids, including capsaicin and four structurally related compounds, namely nordihydrocapsaicin, dihydrocapsaicin, homocapsaicin, and homodihydrocapsaicin (Hoffman et al., 1983). Capsaicin, which is the most abundant capsaicinoid, contributes most to pungency. Quantification of these pungent

Received for publication 24 June 2003. Accepted for publication 11 Mar. 2003. We wish to acknowledge personnel from CARDI, Barbados, who provided the pepper fruit used in this study. We are also grateful to Bertie Linton from Government Analytical Service, Barbados, for his assistance with HPLC determinations and Tanya Edgehill for her part in preparation and extraction of pepper samples.

1E-mail: hgibbs@uwichill.edu.bb compounds is therefore an important index of pepper fruit quality.

Several methods have been developed to assess pungency (Anan et al.,1996; Bajaj, 1980; Krajewska and Powers, 1987; Scoville,1912; Weaver and Awde, 1986). A widely used pungency measurement is the Scoville Unit (Scoville, 1912). This measurement is the highest dilution of pepper extract at which pungency can be detected by a taste panel. Although the Scoville Unit is derived subjectively, it is generally preferred by the spice industry, since other pungency measurements, notably those based on high performance liquid chromatography (HPLC), gas chromatography, and colorimetry, are more time consuming and generally expensive (Todd et al., 1977; Woodbury, 1980). Pungency measurements based on quantification of capsaicinoids are necessary to monitor the reliability of the Scoville method.

Of the methods quantifying capsaicinoids, those based on colorimetry are used most routinely. These methods utilize solvent extraction of capsaicinoids, but chromophores, including chlorophylls, which may interfere with capsaicinoid quantification, are also extracted (Attuquayefio and Buckle, 1987; Palacio, 1979). For this reason, the pepper extract is often prepared to minimize the interference (Bajaj, Cooper et al., 1991; Hoffman et al., 1983;
1980; Jurenitsch and Leinmuller, 1980). The preparation generally requires concentration of pepper extracts by solvent evaporation and removal of interfering compounds by column chromatography. Notwithstanding the popularity of colorimetric methods for capsaicinoid quantification, this preparation is considered a drawback on the basis of time consumption.

The primary objective of this study was to determine levels of pungency of a collection of $C$. chinense grown in the Caribbean. The applicability of a simple colorimetric method, which eliminated solvent evaporation and column chromatographic clean up prior to determination of pungency, was also assessed by comparison with an established method (Bajaj, 1980), which requires pretreatment to remove the interfering substances. Quantitative and qualitative determination of capsaicinoids present in pepper fruit was also done using HPLC and thin layer chromatography, respectively.

\section{Materials and Methods}

Pepper fruit. Mature green and ripe pepper fruit were used in this study. They were obtained from field crops grown at the Experimental Field Station of Caribbean Agricultural Research and Development Institute (CARDI), Barbados. Except for one accession each of Capsicum frutescens and C. annuum, all other fruit were $C$. chinense-types. All accessions are grown in the Caribbean (Table 1).

Apparatus. Colorimetric determination of capsaicinoids was done using a Aku Spec-D spectrometer (Chem-Index, Miami.) of 1$\mathrm{cm}$ cell path length and 5-mL cell capacity. Capsaicinoid content was also determined using a high performance liquid chromatograph (1050 Hewlett Packard Series, Conn.) equipped with UV absorbance detector, a quaternary pump, a Rheodyne injector (710 J syringe loading sample injector) with 089-0930 valve, and 089-0931 loop filler. The separating column was packed with ODS Hypersil of particle size $5 \mu \mathrm{m}$, pore diameter $120 \AA$, pore volume $0.65 \mu \mathrm{L} \cdot \mathrm{g}^{-1}$, surface area $170 \mathrm{~m}^{2} \cdot \mathrm{g}^{-1}$ and carbon loading of $9.1 \%$ to $10.0 \%$ (w/w).

Reagents. HPLC-grade methanol of 99.93\% purity (Aldrich, Wisc.) and ethyl acetate of $99 \%$ purity (BDH, England) were used routinely as solvents in this study. These solvents were degassed and filtered using a 47-mm glass filter holder (Millipore Corp., Mass.) fitted with type HA water filters (Millipore) of $0.45-\mu \mathrm{m}$ pore size, or with type FA methyl alcohol filters (Millipore) of $0.5-\mu \mathrm{m}$ pore size. Double de-ionized water was used in the preparation of all aqueous solutions including $0.5 \mathrm{~N} \mathrm{HCl}$ and $1.0 \mathrm{~N} \mathrm{NaOH}$.

Sample preparation. Sliced green and ripe pepper fruit were dried at $60^{\circ} \mathrm{C}$ for $4-5 \mathrm{~d}$ and ground to a fine powder using a laboratory mill (Christy and Norris, Chelmsford, England). The powder was sifted through a No. 40 sieve (W.S. Tyler, Ohio) and prepared in two ways to assess capsaicinoid content. In one method, dried fruit powder was extracted according to procedures described by Bajaj (1980). In this method, $0.2-\mathrm{g}$ portions of sieved pepper 
Table 1. Capsaicin content of green and ripe pepper fruit representing thirty cultivars of Capsicum sp. grown in the Caribbean.

\begin{tabular}{|c|c|c|c|c|c|c|c|}
\hline \multirow{2}{*}{$\begin{array}{l}\text { Pepper } \\
\text { accession }^{x}\end{array}$} & \multirow[b]{2}{*}{ Species } & \multicolumn{2}{|c|}{ Ripe $(100 \mathrm{mg} / \mathrm{g})^{\mathrm{z}}$} & \multicolumn{2}{|c|}{ Green $(100 \mathrm{mg} / \mathrm{g})^{\mathrm{z}}$} & \multicolumn{2}{|c|}{ Scoville units ${ }^{y}$} \\
\hline & & Bajaj & $\overline{\text { Bajaj}(M)^{v}}$ & Bajaj & Bajaj(M) & ripe & green \\
\hline$\overline{\text { PP-9771951 }}$ & C. chinense & 55.59 vii & 59.59 vii & 52.41 & 53.14 & 45000 & 37500 \\
\hline PP-977122 & C. chinense & $162.8 \mathrm{v}$ & $163.8 \mathrm{iv}$ & 185.0 & 131.1 & 123000 & 97500 \\
\hline PP-977623 $3^{\mathrm{T}}$ & C. chinense & $156.1 \mathrm{vi}$ & $177.8 \mathrm{iv}$ & 136.1 & 130.5 & 135000 & 97500 \\
\hline PP-97421 ${ }^{\mathrm{T}}$ & C. chinense & 281.4 iii & $268.0 \mathrm{ii}$ & 267.7 & 255.7 & 203000 & 195000 \\
\hline PP-9656-02 & C. chinense & 115.4 vi & $113.4 \mathrm{v}$ & 117.1 & 103.2 & 82500 & 75000 \\
\hline PP-977123 & C. chinense & $236.9 \mathrm{iv}$ & 213.4 iii & 189.1 & 158.6 & 158000 & 120000 \\
\hline PP-977644 ${ }^{\mathrm{T}}$ & C. chinense & 142.4 vi & $143.7 \mathrm{iv}$ & 144.7 & 139.4 & 105000 & 105000 \\
\hline PP-977126 & C. chinense & $295.4 \mathrm{iii}$ & $225.2 \mathrm{iii}$ & 297.4 & 219.7 & 173000 & 165000 \\
\hline PP-977127 ${ }^{\mathrm{T}}$ & C. chinense & $198.0 \mathrm{v}$ & $147.0 \mathrm{iv}$ & 171.4 & 133.0 & 113000 & 97500 \\
\hline PP-997197-1 $1^{\mathrm{T}}$ & C. chinense & 54.55 vii & 15.00 vii & 41.38 & 13.68 & 15000 & 7500 \\
\hline PP-977116 & C. chinense & 73.59 vii & 70.32 vii & 85.66 & 46.52 & 52500 & 37500 \\
\hline $\mathrm{PBC} 161^{\mathrm{T}}$ & C. chinense & $192.9 \mathrm{v}$ & 252.7 iii & 174.3 & 166.6 & 188000 & 128000 \\
\hline Barbados Seasoning ${ }^{\mathrm{B}}$ & C. chinense & $101.0 \mathrm{vi}$ & $108.3 \mathrm{vi}$ & 74.72 & 55.26 & 82500 & 45000 \\
\hline St.Lucia Seasoning ${ }^{\mathrm{L}}$ & C. chinense & 37.62 vii & 27.68 vii & 27.82 & 26.67 & 22500 & 22500 \\
\hline Cayenne $^{\mathrm{G}}$ & C. аппиит & 328.6 iii & $257.8 \mathrm{ii}$ & 229.1 & 231.4 & 195000 & 195000 \\
\hline Majaque $^{\mathrm{J}}$ & C. chinense & $280.7 \mathrm{iv}$ & 208.1 iii & 227.9 & 191.3 & 158000 & 143000 \\
\hline Tiger Tooth ${ }^{\mathrm{G}}$ & C. chinense & $257.6 \mathrm{iv}$ & 248.8 iii & 255.2 & 226.3 & 188000 & 173000 \\
\hline Barbados Brown $^{\mathrm{B}}$ & C. chinense & $280.5 \mathrm{iv}$ & $236.1 \mathrm{iii}$ & 281.2 & 216.2 & 180000 & 165000 \\
\hline Touvin ${ }^{\mathrm{GU}}$ & C. chinense & 309.5 iii & $301.9 \mathrm{ii}$ & 297.6 & 291.3 & 225000 & 225000 \\
\hline Red Flat $^{\mathrm{L}}$ & C. chinense & 241.9 vi & 222.6 vi & 238.9 & 138.4 & 165000 & 105000 \\
\hline Bird pepper ${ }^{\mathrm{B}}$ & C. frutescens & 335.1 iii & $236.7 \mathrm{iii}$ & 313.1 & 213.8 & 180000 & 158000 \\
\hline Wirri Wirri (red) ${ }^{\mathrm{G}}$ & C. chinense & $396.1 \mathrm{ii}$ & $289.6 \mathrm{ii}$ & 394.4 & 205.3 & 218000 & 158000 \\
\hline Wirri Wirri (yellow) ${ }^{\mathrm{G}}$ & C. chinense & $401.2 \mathrm{ii}$ & $336.6 \mathrm{i}$ & 377.8 & 333.2 & 255000 & 248000 \\
\hline Caribbean Red ${ }^{\mathrm{A}}$ & C. chinense & $374.3 \mathrm{ii}$ & $293.6 \mathrm{ii}$ & 393.2 & 273.3 & 225000 & 200000 \\
\hline Habanero $^{\mathrm{U}}$ & C. chinense & $497.0 \mathrm{i}$ & $402.4 \mathrm{i}$ & 396.5 & 356.4 & 300000 & 270000 \\
\hline Numex Twilight $^{\mathrm{N}}$ & C. chinense & $75.21 \mathrm{vii}$ & $69.84 \mathrm{vi}$ & 63.80 & 63.34 & 52500 & 45000 \\
\hline W. I. Red (purple) ${ }^{A}$ & C. chinense & $308.1 \mathrm{iii}$ & 223.6 iii & 302.6 & 215.0 & 173000 & 165000 \\
\hline W. I. Red (green) ${ }^{\mathrm{A}}$ & C. chinense & $280.5 \mathrm{iv}$ & $222.3 \mathrm{iii}$ & 286.3 & 245.1 & 168000 & 185000 \\
\hline W. I. Red (cream) ${ }^{\mathrm{A}}$ & C. chinense & $408.5 \mathrm{i}$ & $345.1 \mathrm{i}$ & 404.5 & 326.2 & 260000 & 245000 \\
\hline Yellow Guive ${ }^{\mathrm{GU}}$ & C. chinense & $189.4 \mathrm{v}$ & $185.6 \mathrm{iii}$ & 171.0 & 149.5 & 143000 & 115000 \\
\hline $\operatorname{LSD}_{(0.05)}$ & & 49.38 & 54.79 & 49.38 & 54.79 & & \\
\hline
\end{tabular}

$\mathrm{z}_{\mathrm{i}}$ to vii represent groupings of ripe and green fruit on the basis of decreasing capsaicin content.

${ }^{y}$ Capsaicin content converted to Scoville Units using equation from Palacio (1979).

${ }^{x}$ Superscript letters following names of accessions indicate the source of pepper: A = Antigua; $\mathrm{B}=$ Barbados; $\mathrm{G}=$ Guyana; $\mathrm{GU}=$ Guadeloupe; $\mathrm{J}=$ Jamaica; $\mathrm{L}=$ St. Lucia; $\mathrm{N}=$ New Mexico; $\mathrm{T}=$ Taiwan; and $\mathrm{U}=$ United States.

${ }^{\mathrm{v}} \mathrm{Bajaj}(\mathrm{M})$ represents the modified Bajaj method devised in the present study.

powder were suspended for $24 \mathrm{~h}$ in $5.0 \mathrm{~mL}$ ethyl acetate to extract capsaicinoids. The resultant suspension was filtered through a $0.45-\mu \mathrm{m}$ membrane and the filtrate containing the capsaicinoids extract evaporated to dryness at room temperature. The extract was further treated by column chromatography to purify capsaiconoids as previously described (Bajaj, 1980).

In another method devised during the present study and referred to as the "modified Bajaj method," pepper powder described above was extracted with ethyl acetate following procedures described by Bajaj (1980) except that the filtrate was not allowed to evaporate, but instead mixed with $2.0 \mathrm{~mL}$ $\mathrm{H}_{2} \mathrm{O}$ followed by vigorous shaking. To each mixture was added $4.0 \mathrm{~mL}$ of $0.5 \mathrm{~N} \mathrm{HCl}$ and $2.0 \mathrm{~mL}$ absolute methanol before shaking. The colored organic layer was removed by syringe and discarded and the clear aqueous layer retained and treated with $2.0 \mathrm{~mL}$ sodium nitrite-sodium molybdate reagent.

The ethyl acetate pepper extracts mentioned above were used directly or evaporated to an oily slur before use, using a Buchi Rotavapor R-14 (Brinkman, N.Y.) equipped with water bath, maintained at $35^{\circ} \mathrm{C}$, before analysis using HPLC and TLC. In both methods of sample preparation, ripe and green fruit extracts of 30 pepper cultivars or varieties were prepared in duplicate.
Capsaicinmeasurements (a) Preparation of calibration curve. Standard capsaicin solution was prepared by dissolving $0.0107 \mathrm{~g}$ capsaicin [8-methly- $N$-Vanillyl-6-Nonenamide (Sigma, Mo.) minimum purity of $97 \%$, in $25 \mathrm{~mL}$ ethyl acetate.

Standard solutions for the calibration curve were prepared according to Bajaj (1980) as well as by mixing $0.05,0.10,0.15,0.20,0.25$, $0.30,0.40$, and $0.50 \mathrm{~mL}$ of capsaicin standard

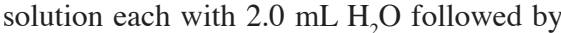
vigorous shaking. To each mixture was added $4.0 \mathrm{~mL}$ of $0.5 \mathrm{~N} \mathrm{HCl}$, followed by $2.0 \mathrm{~mL}$ sodium nitrite-sodium molybdate reagent and then mixed thoroughly. After $15 \mathrm{~min}$. had elapsed and the appropriate color intensity developed, $2.0 \mathrm{~mL} 1 \mathrm{~N} \mathrm{NaOH}$ solution were added and the absorbance determined immediately after at $430 \mathrm{~nm}$. Data from each set of capsaicin standards were used to generate plots of absorbance vs. capsaicin concentration and used as reference for estimating capsaicin content in pepper extract.

\section{Analysis of pepper extract}

Colorimetry. To the extracts prepared according to both Bajaj (1980) and the modified Bajaj method, was added $4.0 \mathrm{~mL}$ of $0.5 \mathrm{~N} \mathrm{HCl}$. For both methods, $2.0 \mathrm{~mL}$ sodium nitrite-sodium molybdate reagent were added and the solutions mixed thoroughly. After $15 \mathrm{~min}$ had elapsed and the appropriate color intensity developed, $2.0 \mathrm{~mL} 1 \mathrm{~N} \mathrm{NaOH}$ solution were added and the absorbance determined immediately at 430 nm using a Aku Spec-D spectrophotometer (Chem-Index, Miami), set at a wavelength of $430 \mathrm{~nm}$. All extracts except those concentrated by the rotovapor were tested by colorimetry. The concentration of capsaicin in all pepper extracts tested was estimated directly from the standard curves. Capsaicin content assessed by the Bajaj method (Bajaj,1980) was converted to Scoville Unit values using the method described by Rajpoot and Govindarajan (1981).

Thin layer chromatography. Thin layer chromatography conducted on 60 F254 silica gel plates (Aldrich, Germany) of layer thickness $0.25 \mathrm{~mm}$ was used to visualize the interfering pepper extract pigments, and to evaluate the effectiveness of treatment to minimize the interference. Ethyl acetate pepper extracts previously concentrated using the rotovapor as described before and samples of commercial capsaicin were spotted and concentrated onto silica gel chromatography plates using a capillary tube. Extracts previously obtained from spinach (Bassela alba) using the Bajaj method(Bajaj, 1980) without the clean-up step by column chromatography and concentrated by the rotovapor as described before for pepper extracts were similarly prepared for thin layer chromatography.

The chromatogram was developed at $20^{\circ} \mathrm{C}$ using a 1 ethyl acetate : 4 hexane mixture as eluant. $\mathrm{Rf}$ values of eluates were determined following treatment with iodine and visualization under ultraviolet light. Spinach was used as a reference to identify chlorophylls and carotenoids, all of which have been previously characterized by their $\mathrm{Rf}$ values (Boliger and Konig, 1969).

High performance liquid chromatography HPLC was performed on ethyl acetate pepper extracts prepared by the Bajaj method (Bajaj, 1980) and on dilutions of the standard capsaicin (Sigma) prepared from $0.428 \mathrm{~g} \cdot \mathrm{L}^{-1}$ stock. The HPLC was run for 6 min using a flow rate of $1 \mathrm{~mL}$ per minute, maximum pressure of 300 bars, $\mathrm{pH}$ range of $2-8$, and a temperature of 28 to $30^{\circ} \mathrm{C}$. Three, $25-\mu \mathrm{L}$ portions of each sample were injected into the port and eluted in a solvent (mobile phase) made up of $70 \%$ methanol and $30 \%$ of a $10 \%$ methanol solution. All solvents were filtered through $0.45-\mu \mathrm{m}$ pore size filters, and degassed immediately before using. The attenuation was set at 6 .

Relative retention times and peak areas of the five capsaicinoids present in commercial capsaicin were determined according to methods described by Cooper et al. (1991) and used to quantify the capsaicinoids found in pepper extracts.

\section{Results and Discussion}

Capsaicin content, as determined by the method outlined by Bajaj (1980), was generally higher in ripe fruit than green fruit (see Table 1). The content ranged from 37.6 $-497.0 \mathrm{mg} / 100 \mathrm{~g}$ in ripe fruits and $27.8-404.5$ $\mathrm{mg} / 100 \mathrm{~g}$ in green fruits. The modified Bajaj 
method produced ranges of capsaicin content of $15.0-402.4 \mathrm{mg} / 100 \mathrm{~g}$ and $13.7-356.4 \mathrm{mg} /$ $100 \mathrm{~g}$ for ripe and green fruits respectively. The Bajaj method invariably gave higher levels of capsaicin than the modified Bajaj method for all extracts except 'Cayenne' and PP-9771951, both of which were lower in capsaicin content. Of the two methods, the Bajaj procedure also gave significantly higher $(P \leq 0.05)$ mean capsaicin content.

On the basis of capsaicin content assessed by colorimetry, the pepper cultivars were differentiated into seven significantly different groups (Table 1). For the modified Bajaj method, groupings of cultivars on the basis of increasing levels of capsaicin in ripe or green fruit are as follows: 'St. Lucia Seasoning', PP-9771951, PP-997197-1, and PP-977116 (Group VII); 'Barbados Seasoning' and 'Numex Twilight' (Group VI); PP-9656-02 (GroupV); PP-977122, PP977623, PP977644, PP-977127, and 'Red Flat' (Group IV); PP-977123, PP-977126, PBC161, 'Majaque', 'Tiger Tooth', 'Barbados Brown', 'Bird Pepper', 'West Indies Red' (purple), 'West Indies Red' (green), and 'Yellow Guive' (Group III); 'Caribbean Red', 'Wirri Wirri' (red), 'Touvin', 'Cayenne', and PP-97421 (Group II); and 'West Indies Red' (cream), 'Habanero', and 'Wirri Wirri' (yellow) (Group I).

Similar analysis of capsaicin content of ripe or green fruit by the Bajaj method was also used to place the cultivars into seven different pungency groups. Groups of the same numerical assignment for each method of capsaicin assessment were mainly comprised of common pepper accessions.

Scoville measurements of pungency derived from capsaicin levels obtained from the Bajaj method are shown in Table 1. These pungency measurements for the accessions varied from 15,000 to 300,000 Scoville units for ripe fruit and 7,500 to 270,000 Scoville units for green fruit. The extent to which these measurements actually represent pungency assessment by a taste panel has not been determined in the present study.

Proof of the possible adverse effects of pigments on colorimetric determination of capsaicin content of pepper fruit was provided by the evidence obtained from TLC conducted on extracts of both green and ripe pepper fruit, spinach, and commercial capsaicin. One detectable pigment was associated with green pepper, while many, ranging in color from pink through orange and yellow to green, were associated with ripe pepper (Table 2). One UV sensitive spot only of Rf 0.091 was associated with pure capsaicin, and corresponded to a similar colorless spot each in the chromatogram of green and ripe pepper extracts. Results in Table 2 also show that, apart from the orange pigment of $\mathrm{Rf}$ 0.036 and the green pigment of Rf 0.014 present in extracts of ripe and green pepper fruit respectively, all other extracted pigments were significantly transported in the eluting solvent. This suggests that capsaicin present in pepper fruit is more polar than most of these pigments and therefore can be separated on the basis of its polarity.
Table 2. Rf values of eluates on thin layer chromatography ethyl acetate extracts of spinach, ripe and green pepper fruit and commercial capsaicin using 1 ethyl acetate : 4 hexane as eluting solvent.

\begin{tabular}{lccccc}
\hline & & \multicolumn{3}{c}{ Color of spots observed for plant extracts and standard } \\
\cline { 3 - 6 } Rf & Likely compound & Spinach & Ripe pepper & Green pepper & Capsaicin $^{2}$ \\
\hline 0.014 & chlorophyll & $---y$ & --- & green & --- \\
0.036 & carotenoid & --- & orange & --- & --- \\
0.091 & capsaicin & --- & colorless & colorless & colorless \\
0.117 & chlorophyll & dark green & -- & --- & --- \\
0.179 & vitamin & --- & yellow & --- & --- \\
0.219 & vitamin & --- & light pink & --- & --- \\
0.321 & vitamin & --- & orange & --- & --- \\
0.365 & chlorophyll & green & --- & --- & --- \\
0.467 & vitamin & --- & orange & --- & --- \\
0.482 & chlorophyll & dark green & --- & -- & --- \\
0.657 & xanthophyll & --- & yellow & --- & --- \\
0.701 & chlorophyll & dark green & --- & --- & --- \\
0.825 & xanthophyll & --- & yellow & --- & --- \\
0.927 & vitamin & --- & pink & --- & --- \\
0.956 & carotenoid & --- & orange & -- & --- \\
0.971 & carotenoid & light orange & light orange & --- & --- \\
\hline
\end{tabular}

${ }^{2}$ Capsaicin standard, minimum assay $97 \%$.

${ }^{y}$ No corresponding spot on the TLC plate.
Treatment of ethyl acetate pepper extract with acidified aqueous solution resulted in a thin, bright orange organic layer above a colorless aqueous solution, which gave no absorbance in the visible region of the spectrum (data not shown). This observation suggests that possibly the pigmented constituents of pepper extract could by separated from capsaicin by aqueous treatment following which the latter could be determined via colorimetric analysis.
The method of using acidified aqueous solution to separate interfering chromogenic pigments from capsaicin to minimize the interference in colorimetric determination of capsaicin content of pepper extract is a key feature of the modified Bajaj method of capsaicin assessment devised in the present study. Unlike the Bajaj method, the extracting solvent was not removed. Conclusions drawn from data obtained for each method were generally similar(Table 1), but the Bajaj procedure

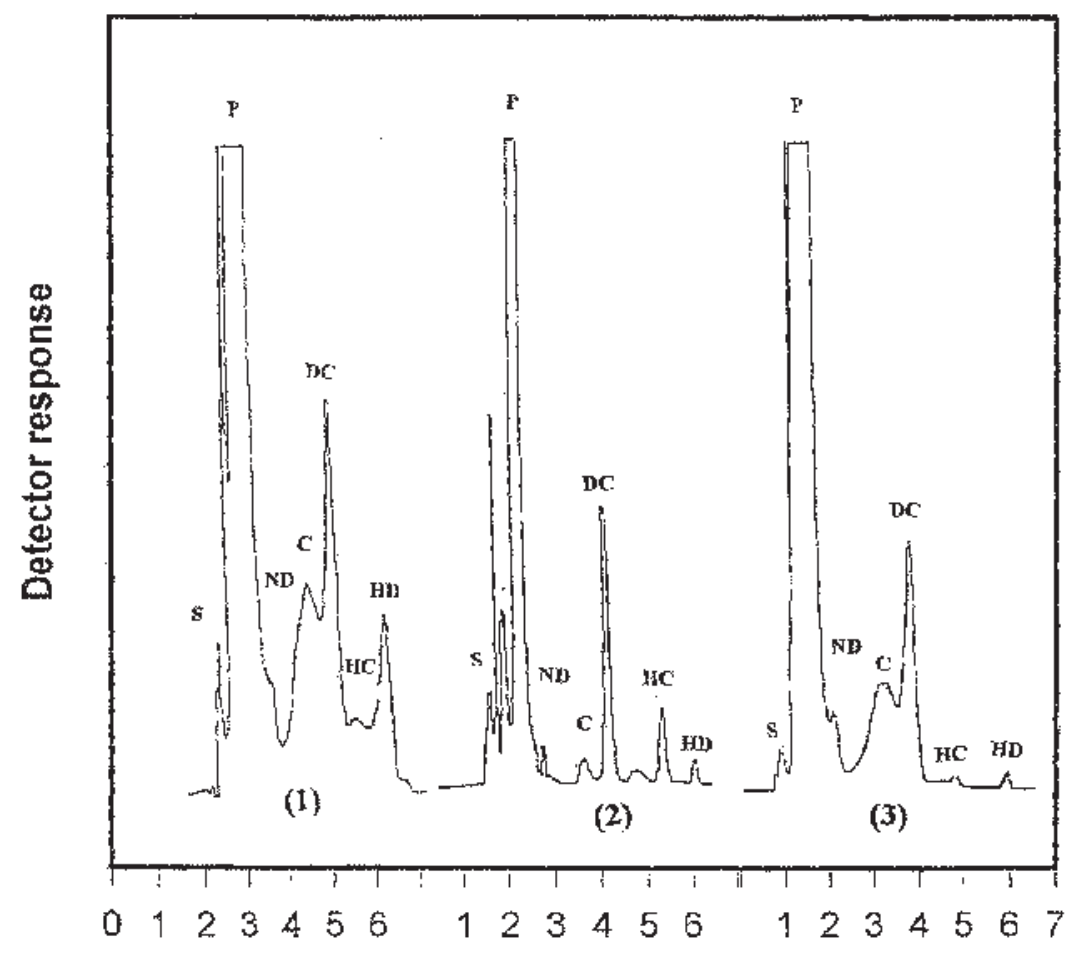

Retention time (mìn.)

Fig. 1. HPLC UV detection chromatograms of: (1) Capsicum chinense ripe fruit; (2) Capsicum chinense green fruit; and (3) capsaicin, showing separation of capsaicinoids and pigments for a 6-min run (short run) using: Column, Zorbax SB - C18; sample size, $25 \mu \mathrm{L}$; flow rate, $0.75 \mathrm{~mL} / \mathrm{min}$; solvent, methanol/ 10\% methanol (70:30). Illustrated are: Solvent (s); pigment (p); nordihydrocapsaicin (ND); capsaicin (C); dihydrocapsaicin (DC); homocapsaicin (HC); and homodihydrocapsaicin (HD). 
Table 3. Capsaicinoid content $(100 \mathrm{mg} / \mathrm{g})$ obtained for green and ripe pepper fruit harvested from among the West Indian grown pepper collection using HPLC UV detection.

\begin{tabular}{|c|c|c|c|c|c|c|}
\hline Pepper accession & Species & ND & $\mathrm{C}$ & $\mathrm{DC}$ & $\mathrm{HC}$ & HD \\
\hline PP-9771951 & C. chinense & $-(-)^{\mathrm{z}}$ & $178(149)$ & $27(1)$ & $390(7)$ & $3(-)$ \\
\hline PP-977122 & C. chinense & 7 (10) & $540(37)$ & $2(-)$ & $60(9)$ & $-(-)$ \\
\hline PP-977623 & C. chinense & 1 (4) & 27 (9) & $2(-)$ & $15(-)$ & $-(3)$ \\
\hline PP-97421 & C. chinense & $4(2)$ & 316 (227) & $11(6)$ & $156(47)$ & $-(-)$ \\
\hline PP-9656-06 & C. chinense & $5(4)$ & $175(-)$ & 7 (3) & $20(32)$ & 7 (38) \\
\hline PP-977123 & C. chinense & $5(2)$ & $363(3)$ & $13(1)$ & $183(12)$ & $-(3)$ \\
\hline PP-977644 & C. chinense & $1(6)$ & $62(8)$ & $2(1)$ & $16(6)$ & $-(1)$ \\
\hline PP-977126 & C. chinense & $-(2)$ & $23(10)$ & $2(2$ & $23(15)$ & $-(-)$ \\
\hline PP-977127 & C. chinense & 4 & 112 & 3 & 46 & 14 \\
\hline PP-997197-1 & C. chinense & $1(3)$ & $325(198)$ & $7(5)$ & $42(-)$ & $207(-)$ \\
\hline PP-977116 & C. chinense & $2(5)$ & 267 (12) & $7(-)$ & 109 (27) & $-(20)$ \\
\hline $\mathrm{PBC} 161$ & C. chinense & 6 & 93 & 3 & 39 & - \\
\hline Barbados Seasoning & C. chinense & $-(1)$ & $164(2)$ & $5(2)$ & $58(-)$ & $3(-)$ \\
\hline St.Lucia Seasoning & C. chinense & $-(5)$ & $34(22)$ & 1 (1) & $2(5)$ & $-(10)$ \\
\hline Cayenne & C. annuит & $-(4)$ & $579(375)$ & $14(9)$ & $72(1)$ & 19 (129) \\
\hline Majaque & C. chinense & $8(4)$ & $290(14)$ & $1(3)$ & $53(24)$ & $12(-)$ \\
\hline Tiger Tooth & C. chinense & $5(4)$ & 513 (127) & $13(5)$ & $104(29)$ & $1(-)$ \\
\hline Barbados Brown & C. chinense & $4(2)$ & $730(350)$ & $35(10)$ & $140(64)$ & $-(-)$ \\
\hline Touvin & C. chinense & 5 & 396 & 12 & 97 & - \\
\hline Red Flat & C. chinense & $1(1)$ & $91(7)$ & $4(1)$ & $23(6)$ & $-(10)$ \\
\hline Bird pepper & C. frutescens & $6(5)$ & 357 (273) & $11(7)$ & $178(127)$ & $12(-)$ \\
\hline Wirri Wirri (red) & C. chinense & $2(-)$ & $181(110)$ & $5(3)$ & $13(28)$ & $198(-)$ \\
\hline Wirri Wirri (yellow) & C. chinense & $4(1)$ & $212(179)$ & 7 (4) & $65(90)$ & 4 (18) \\
\hline Caribbean Red & C. chinense & $5(1)$ & $313(95)$ & $11(6)$ & $117(24)$ & $-(19)$ \\
\hline Habanero & C. chinense & $4(5)$ & $339(-)$ & $10(2)$ & $39(25)$ & $45(-)$ \\
\hline Numex Twilight & C. chinense & $\begin{array}{ll}5 & 4)\end{array}$ & $265(65)$ & $3(3)$ & $71(43)$ & $5(8)$ \\
\hline W. I. Red (purple) & C. chinense & $6(-)$ & $490(181)$ & $4(5)$ & $93(46)$ & $-(-)$ \\
\hline W. I. Red (green) & C. chinense & $-(-)$ & $1650(84)$ & $5(2)$ & $245(27)$ & $4(-)$ \\
\hline W. I. Red (cream) & C. chinense & $-(-)$ & $283(129)$ & $11(3)$ & $71(46)$ & $-(-)$ \\
\hline Yellow Guive & C. chinense & $3(5)$ & $178(42)$ & $5(1)$ & $38(14)$ & $-(8)$ \\
\hline
\end{tabular}

${ }^{2}$ Parentnesis indicate capsaicinoid content of green pepper fruit.

y - indicates No capsaicinoids detected using HPLC UV detection.

$(\mathrm{ND})=$ nordihydrocapsaicin; $(\mathrm{C})=$ capsaicin; $(\mathrm{DC})=$ dihydrocapsaicin; $(\mathrm{HC})=$ homocapsaicin; $(\mathrm{HD})=$ homodihydrocapsaicin .

is more time-consuming on the basis of the intervention of steps to remove the solvent by evaporation and interfering pigments by column chromatography.

Quantitative and qualitative assessment of capsaicinoids in pepper fruit using HPLC is based on the unique retention time and peak size of each capsainoid present in varying concentrations of commercially available capsaicin as well as in pepper extracts. The capsaicinoids, including, nordihydrocapsaicin, capsaicin, dihydrocapsaicin, homocapsaicin, and homodihydrocapsaicin, were detected at 2.5, 3.2, 4.0, 5.2, and $6.1 \mathrm{~min}$, respectively. These retention times observed for the five capsaicinoids present in pepper extract were similar to those observed by Cooper

\section{Literature Cited}

American Spice Trade Association (ASTA). 1985. Official analytical methods of the ASTA. 3rd ed. ASTA., Englewood Cliffs. N.J.

Anan, T., H. Ito, H. Matsunaga, and S. Monma. 1996. A simple method for determining the degree of pungency of peppers. Capsicum and Eggplant Nwsl. 15:51-54.

Attuquayefio, V.K. and K.A. Buckle. 1987. Rapid sample preparation method for HPLC analysis of Capsicum fruits and oleoresins. J. Agr. Food Chem. 35:777-779.

Bajaj, K.L. 1980. Colorimetric determination of capsaicin in Capsicum fruits. J. Assoc. Offic. Anal. Chem. 63(6):1314-1316.

Bolliger, H.R. and A. Konig, (1969). K vitamins, including carotenoids, chlorophlyys, and biologically active quinones, p. 259-308. In: E. Stahl, (ed.). Thin-layer chromatography; A laboratory handbook. Springer-Verlag, N.Y.

Cooper, T.H., J.A. Guzinski, and C. Fisher. 1991. Improved HPLC method for the determination of major capsaicinoids in Capsicum oleoresins. J. Agr. Food Chem. 39:2253-2256.

Hoffman, P.G., M.C. Lego, and W.G. Galetto. 1983. Separation and quantitation of red pepper major heat principles by reverse-phase HPLC. J. Agr. Food Chem. 31:1326-1330.

Jurenitsch, J. and R. Leinmuller. 1980. Quantification of nonylic acid vanillylamide and other capsaicinoids in the pungent principle of Capsicum fruits and preparation by GLC on glass capillary columns. J. Chromatogr. 189:389.

Krajewska, A.M. and J.J. Powers. 1988. Sensory properties of naturally occuring capsaicinoids. J. Food Sci. 53:902-905.

Palacio, J.J.R. 1979. Further study of the spectrophotometric determination of capsaicin. J.Assoc. Offic. Anal. Chem. 62(5):1168-1170.

Rajpoot, N.C. and V.S. Govindarajan. 1981. Paper chromatographic determination of total capsaicinoids in capsicums and their oleoresins with precision, reproducibility and validation through correlation with pungency in Scoville Units. J. Assoc. Off. Anal. Chem. 64(2):311-318.

Scoville, W.L. 1912. Note Capsicum. J. Amer. Pharm. Assn. 1:453.

Todd, P. H., Bensinger and M.T. Biftu. 1977. Determination of pungency due to Capsicum by GLC. J. Food Sci. 42:660

Weaver, K.M. and D.B. Awde. 1986. Rapid HPLC method for the determination of very low capsaicin levels. J. Chromatogr. 367:438-442.

Woodbury, J.E. 1980. Determination of Capsicum pungency by HPLC and spectrofluorometric detection. J. Assn. Offic. Anal. Chem. 63(3): $556-558$. 